\title{
A Method of Moments Approach for Laminar Boundary Layer Flows
}

\author{
Omer Kemal Kinaci ${ }^{1^{*}}$ and Onur Usta ${ }^{2}$ \\ I Yildiz Technical University, Faculty of Naval Architecture and Maritime, Istanbul, 34349, Turkey \\ 2 Istanbul Technical University, Faculty of Naval Architecture and Ocean Engineering, Istanbul, 34469, Turkey
}

(Manuscript Received June 12 2013; Revised July 15, 2013; Accepted August 12, 2013)

\begin{abstract}
Blasius equation describes the boundary layer formed over a flat plate inside a fluid and this equation is solved numerically by the method of moments which is a type of weighted residual methods. Compared to the traditionally used Runge - Kutta Method, Method of Moments propose a direct solution to Blasius Equation which makes it easier to solve. The obtained solutions show good agreement with the results found in literature and this study aims to demonstrate the power of the method.
\end{abstract}

Keywords: Blasius equation, boundary layer theory, method of moments, Falkner-Skan equation

\section{Introduction}

It is one of the mostly used procedures to calculate the viscous flow around objects in fluid flow by coupling the potential flow with the boundary layer theory. A general procedure consists of solving the potential flow around the object by the boundary element method and making the viscous corrections by Blasius equation.

Prandtl first time presented the boundary layer theory in 1904 which was originally presented for laminar flow [1]. The theory suggests that the velocity on the surface of a stationary body is equal to zero and becomes equal to the outer flow velocity over a thin layer called the boundary layer. The theory is accepted by scientific societies and helped optimizing the design of aircrafts, marine craft and other equipment using fluid flow. The boundary layer theory is now commonly used and implemented in many fields of science and engineering.

Blasius equation represents the boundary layer on a flat plate and is derived from the momentum *Corresponding author. Tel.: +90-212-3833015, Fax.: +90-212-3833021 E-mail address: kinaci@yildiz.edu.tr Copyright (C) KSOE 2013. equation. There are some analytical solutions offered to it [2-5]; however they contain approximations just like numerical methods do. It is a form of the Falkner - Skan equations representing the laminar wedge flows. A special case of wedge flow arises when the infinitely thin plate is placed parallel to the incoming flow giving rise to Blasius equation.

There are many numerical methods presented in the literature to solve the equation numerically. The pioneering work of Howarth has proved to be the first attempt in solving the laminar boundary layer equations by Runge - Kutta method [6]. An approximate solution using the homotopy perturbation method was preferred by Filobello - Nino et al. [7] while Paranda et al. has suggested to use the sinc collocation method [8]. Coupled method combining the iteration and the perturbation methods was used by He to obtain high accuracy of the equation [9]. Benlahsen et al. have applied Blasius equation for the solution to non - Newtonian fluids while proving the existence and uniqueness of the results [10]. Cortell has extended the work to a more generalized form of the equation [11] and Fang et al. suggested 
that the extended form shows temperature dependent property of the fluid flow over a flat plate [12].

There are also numerical solutions presented to the more generalized Falkner - Skan equations. The solutions and dependence on wedge angle were investigated by Hartree [13]. Rao has used two methods which were Galerkin and least squares methods to calculate the velocity profiles over a flat plate [14]. Asaithambi has plenty of works on the numerical solutions of the Falkner - Skan equation. He has used the first [15] and second [16] order finite difference methods, piecewise linear equations [17] and recursive evaluation of Taylor coefficients [18].

This study proposes another method which is the method of moments and aims to show the robustness of the method in dealing with ordinary differential equations. As it is widely known; before applying Runge - Kutta Method, a starting point for the iterative solution must be maintained by shooting method while Method of Moments offer a direct solution to the problem. The method proposed in this paper to numerically solve Blasius equation is applied for the first time in the literature.

\section{Derivation of Blasius Equation}

Blasius equation is a special form of the Falkner Skan equation which approximates the boundary layer formation over wedge flows. Falkner - Skan equation is given as:

$$
f^{\prime \prime \prime}+f f^{\prime \prime}+\beta\left(1-f^{\prime 2}\right)=0
$$

Here, $\beta$ is the angle which the wedge makes with the axis of the flow. If the wedge is parallel to the flow, then $\beta=0$ and the resulting equation is called the famous Blasius equation. Blasius equation therefore gives the boundary layer formation over flat plates and is frequently used for viscous corrections to potential flow solutions. A brief derivation of Blasius equation is given below.

A steady two dimensional Prandtl boundary layer equations for a flat plate given in figure 1 are given as:

$\frac{\partial u}{\partial x}+\frac{\partial v}{\partial y}=0$

$u \frac{\partial u}{\partial x}+v \frac{\partial u}{\partial y}=-\frac{1}{\rho} \frac{\partial \rho}{\partial x}+\vartheta \frac{\partial^{2} u}{\partial y^{2}}$ $0=-\frac{\partial \rho}{\partial y}$

Outer flow does not change vertically and the momentum equation for the outer flow reduces to:

$U \frac{d U}{d x}=-\frac{1}{\rho} \frac{\partial \rho}{\partial x}$

For y component of the momentum equation, substitution of eqn. (5) into eqn. (3) gives:

$u \frac{\partial u}{\partial x}+v \frac{\partial u}{\partial y}=U \frac{d U}{d x}+\vartheta \frac{\partial^{2} u}{\partial y^{2}}$

Let the stream function be defined as;

$\varphi=f(\eta) \sqrt{2 \vartheta U x}$

Here $\eta$ is the dimensionless variable used instead of the $\mathrm{y}$ - component of the subjected flow. Presentation of the velocity components in terms of the stream function brings;

$u=\frac{\partial \varphi}{\partial y}$ and $v=-\frac{\partial \varphi}{\partial x}$

where y momentum equation can be rewritten as:

$\frac{\partial \varphi}{\partial y} \frac{\partial^{2} \varphi}{\partial x \partial y}-\frac{\partial \varphi}{\partial x} \frac{\partial^{2} \varphi}{\partial y^{2}}=U \frac{d U}{d x}+\vartheta \frac{\partial^{3} \varphi}{\partial y^{3}}$

Substitution of eqn. (7) into eqn. (9) and rearranging the resulting equation after some transformations brings:

$f^{\prime \prime \prime}(\eta)+f(\eta) f^{\prime \prime}(\eta)=0$

Eqn. (10) is named as the Blasius equation and is widely used for determining the laminar boundary layer flows along flat plates. For wider explanation of the theory, reference [19] could be referred to.

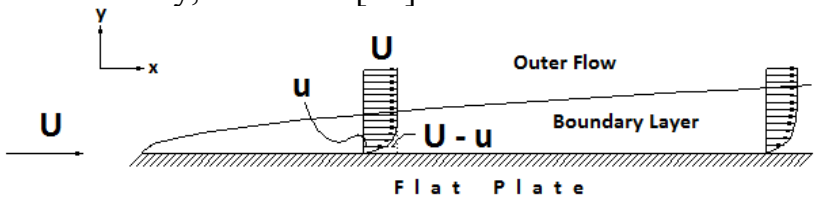

Figure 1. Representation of the flow over a flat plate 
3. Solution Procedure with the Method of Moments

The method of moments is a subset of the weighted residual methods and is generally used in numerical electromagnetic studies. The main idea of the method of moments is to set the residual error to zero through prescribed conditions. The method is generally applied in five steps which may be summarized as:

1. The trial function is stated with unknown coefficients.

2. Boundary and initial conditions are used to satisfy the trial functions.

3. Residual is defined.

4. Weighted residual is set equal to zero and the solutions to the equations are obtained.

5. After successive approximations, the error is examined and convergence is achieved when the desired error is caught.

A function of the form by a polynomial degree $n$ with respect to Weierstrass approximation theorem could be stated as;

$$
P(x)=a_{n} x^{n}+a_{n-1} x^{n-1}+\cdots+a_{1} x+a_{0}
$$

where $a_{n}$ is the leading coefficient. Eqn. (11) is called a genuine $\mathrm{n}^{\wedge}$ th degree polynomial and according to the theorem every continuous function can be approximated in an interval $[\mathrm{a}, \mathrm{b}]$.

The Blasius equation concerning the boundary layer flow over a flat plate is approximated using Weierstrass approximation theorem and the trial function $f(\eta)$ is defined as a eighth degree polynomial:

$$
\begin{aligned}
f(\eta)= & a+b \eta+c \eta^{2}+d \eta^{3}+e \eta^{4}+g \eta^{5} \\
& +h \eta^{6}+i \eta^{7}+j \eta^{8}
\end{aligned}
$$

Blasius equation involves the first, second and the third derivatives of the trial function. Taking these derivatives of $f(\eta)$ will bring the equations:

$$
\begin{aligned}
f^{\prime}(\eta)= & b+2 c \eta+3 d \eta^{2}+4 e \eta^{3}+5 g \eta^{4} \\
& +6 h \eta^{5}+7 i \eta^{6}+8 j \eta^{7} \\
f^{\prime \prime}(\eta)= & 2 c+6 d \eta+12 e \eta^{3}+20 g \eta^{3} \\
& +30 h \eta^{4}+42 i \eta^{5}+56 j \eta^{6} \\
f^{\prime \prime \prime}(\eta)= & 6 d+24 e \eta+60 g \eta^{2} \\
& +120 h \eta^{3}+210 i \eta^{4}+336 j \eta^{5}
\end{aligned}
$$

The unknown parameters $\mathrm{a}$ and $\mathrm{b}$ can be found by applying the boundary conditions;

$$
\begin{aligned}
& \eta=0: f(\eta)=0 \rightarrow a=0 \\
& \eta=0: f^{\prime}(\eta)=0 \rightarrow b=0
\end{aligned}
$$

The last boundary condition states that the velocity of the flow over the flat plate becomes equal to the outer flow velocity as the dimensionless vertical parameter $\eta$ goes to infinity. To satisfy the trial function with the infinity boundary condition, $\eta$ is selected to be equal to 5 . So the last boundary condition becomes:

$\eta=5: f^{\prime}(\eta)=1$

Following the solution procedure, the residual must be defined. The residual $R(\eta)$ is set to be:

$$
R(\eta)=f^{\prime \prime \prime}(\eta)+f(\eta) * f^{\prime \prime}(\eta)
$$

Weighted functions are defined as power of independent variation (or variations) in the method of moments.

$$
\begin{aligned}
& w_{n}=\eta^{n-1} ; n=1,2,3, \ldots, N \\
& \int_{\eta} w_{n} R d \eta=\int_{\eta} R \eta^{n-1} d \eta=0 ; n=1,2,3, \ldots, N
\end{aligned}
$$

The method of moments forces the residual to zero when weighted by increasingly higher members of a complete set of functions, so as $\mathrm{N}$ becomes large the residual must approach to zero. [20] 


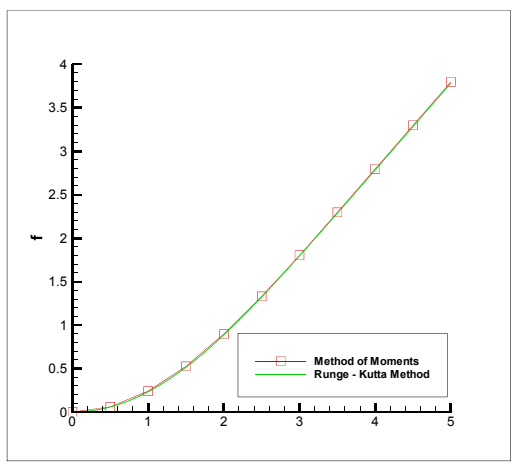

Fig.2. Comparison of the method of moments with the Runge - Kutta method for $f(\eta)$

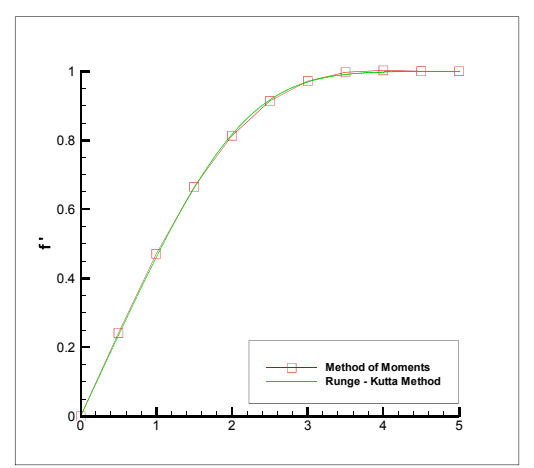

Fig. 3. Comparison of the method of moments with the Runge - Kutta method for $f^{\prime}(\eta)$.

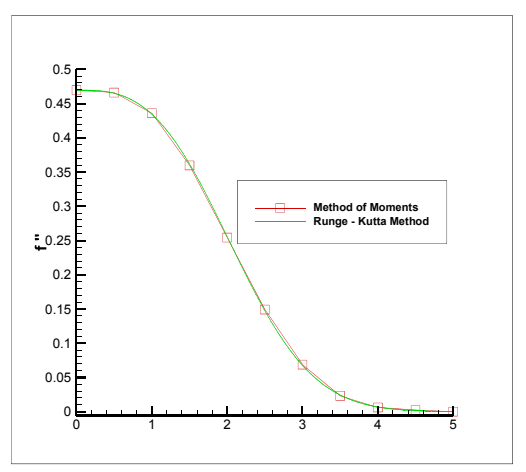

Fig. 4. Comparison of the method of moments with the Runge - Kutta method for $f^{\prime \prime}(\eta)$.

\section{Comparison of the Results}

Boundary layer over a flat plate in uniform flow is solved with the method of moments which is one of the weighted residual methods. The solutions are compared with the fourth order Runge - Kutta integration used together with the shooting method which is a frequent solution procedure used to solve the Blasius equation. The obtained results for $f(\eta)$, $f^{\prime}(\eta)$ and $f^{n}(\eta)$ are given in figures 2, 3 and 4 respectively.

Analyzing the figures, it could be stated that quite good agreements are found for all $f(\eta)$ and its derivatives. It could be seen that as $\eta$ goes to infinity the outer flow velocity is grasped. Higher accuracy can be obtained at higher trial function degrees. However for such higher precision solutions, the computation time must be taken into consideration.

The tabular version of the graphics given in the figures can be seen from table 1 .

\section{Conclusion}

Blasius equation is a third order nonlinear ordinary differential function which represents the viscous fluid flow over a flat plate with a laminar boundary layer. There are lots of ways to solve this equation numerically as per open literature, with many books preferring to publish the approximated solution using Runge - Kutta method. This study approaches to solve Blasius equation with the Method of Moments which is one of the weighted residual methods. Method of moments is easy to implement and proposes a wide range of study fields. Compared to the traditionally used Runge Kutta Method, Method of Moments initiates a faster solution. The goal of this study is to demonstrate the power of the method of moments which is not a frequently used method in aero / hydromechanics. Despite the less interest shown in the method of moments, this study proves that it offers a powerful tool to accurately calculate the velocity profile over a flat plate. The authors' opinion is that this method could be applied to numerically solve many other problems in mechanics involving fluid flow.

\section{Acknowledgment}

The authors are indebted to Prof. Muhittin Soylemez.

\section{References}

[1] I. Tani, History of boundary-layer theory, Ann. Rev. Fluid Mech. 9 (1977) 87-111.

[2] H. Aminikhah, An analytical approximation for solving nonlinear Blasius equation by NHPM, Numerical Methods for Partial Differential Equations, 26 (6) (2010) 1291-1299

[3] J. H. He, Approximate analytical solution of Blasius' equation, Communications in Nonlinear Science \& Numerical Simulation, 4 (1) (1999) 75-78

[4] B. K. Datta, Analytic solution for the Blasius equation, Indian Journal of Pure and Applied Mathematics, 34 (2) (2003) 237-240

[5] L. Wang, A new algorithm for solving classical Blasius equation, Appl. Math. Comput. 157 (2004) 1-9

[6] L. Howarth, On the solution of the laminar boundary layer equations, Proc. Roy. Soc. London A 164 (1938) 547-579.

[7] U. Filobello-Nino, H. Vazquez-Leal, R. Castaneda-Sheissa, A. Yildirim, L. HernandezMartinez, D. Pereyra-Diaz, A. Perez-Sesma, C 
Table 1. Numerical values obtained with the method of moments.

\begin{tabular}{cccc}
$\eta$ & $f(\eta)$ & $f^{\prime}(\eta)$ & $f^{\prime \prime}(\eta)$ \\
\hline 0 & 0.000000 & 0.000000 & 0.469571 \\
0.5 & 0.058501 & 0.233828 & 0.466071 \\
1 & 0.232894 & 0.461266 & 0.436032 \\
1.5 & 0.515294 & 0.661945 & 0.359704 \\
2 & 0.887003 & 0.816067 & 0.254326 \\
2.5 & 1.322310 & 0.916356 & 0.149257 \\
3 & 1.795454 & 0.969520 & 0.068859 \\
3.5 & 2.286560 & 0.991090 & 0.023152 \\
4 & 2.784057 & 0.997526 & 0.006215 \\
4.5 & 3.283380 & 0.999467 & 0.002356 \\
5 & 3.783300 & 1.000000 & 0.000033 \\
\hline
\end{tabular}

Hoyos-Reyes, An approximate solution of Blasius equation by using HPM method, Asian Journal of Mathematics \& Statistics, 5 (2) (2012) 50-59

[8] K. Parand, M. Dehghan, A. Pirkhedri, Sinccollocation method for solving the Blasius equation, Physics Letters A, 373 (44) (2009) 4060-4065

[9] J. H. He, A simple perturbation approach to Blasius equation, Applied Mathematics and Computation, 140 (2-3) (2003) 217-222

[10]M. Benlahsen, M. Guedda, R. Kersner, The generalized Blasius equation revisited, Mathematical and Computer Modelling, 47 (9-10) (2008) 1063-1076
[11]R. Cortell, Numerical solutions of the classical Blasius flat-plate problem, Appl. Math. Comput. 170 (1) (2005) 706-710

[12]T. Fang, F. Guob, C. F. Leea, A note on the extended Blasius equation, Applied Mathematics Letters, 19 (7) (2006) 613-617

[13]Hartree, D. R., On an equation occurring in Falkner and Skan's approximate treatment of the equations of the boundary layer, Proc. Cambridge Philos. Society 33 (1937) 223-239.

$[14]$ G.V. Rao, Least square and galerkin finite element solution of flow past a flat plate, Int. J. for Numer. Meth. Engng. 11 (1) (1975) 185-190.

[15]A. Asaithambi, A finite-difference method for the Falkner-Skan equation, Appl. Math. Comput. 92 (1998) 135-141.

[16]A. Asaithambi, A second-order finitedifference method for the Falkner-Skan equation, Appl. Math. Comput. 156 (2004) 779-786.

[17]A. Asaithambi, Numerical solution of the Falkner-Skan equation using piecewise linear equation, Appl. Math. Comput. 159 (2004) 267-273.

[18]A. Asaithambi, A solution of the Falkner-Skan equation by recursive Taylor coefficients, Journal of Computational and Applied Mathematics, 176 (2005) 203-214.

[19]H. Schlichting, K. Gersten, Boundary Layer Theory, eighth rev.ed., McGraw-Hill, New York, 1999.

[20]B. A. Finlayson, Nonlinear Analysis in Chemical Engineering, McGraw Hill, New York, 1980 